\title{
Comparative Study on Control Method for Two-Mass Systems
}

\author{
Mohd Fitri Mohd Yakub ${ }^{\#}$, Abdul Qadir ${ }^{*}$, B.A.Aminudin ${ }^{\#}$ \\ ${ }^{*}$ Department of Mechanical Engineering, Malaysia-Japan International Institute of Technology, \\ Universiti Teknologi Malaysia International Campus, Kuala Lumpur, Malaysia \\ E-mail: irtif_81@yahoo.com,aminuddin@ic.utm.my \\ *Department of Mechatronic Engineering, International Islamic University Malaysia, Kuala Lumpur, Malaysia \\ E-mail: aqadir101@gmail.com
}

\begin{abstract}
This paper presents study review on control method for two-mass systems either in speed control or position control. The systems should be modeled as two-mass or multi-mass systems when flexible couplings or long shaft with low stiffness are used to connect the actuator with other elements. Due to this, the finite but small elasticity of the shaft gets magnified and has a vibrational effect on the load position which may reduce positioning accuracy. This vibration gives the unstable performance response of the plant. Many control method have been developed to solve this problem are studied. On the other hand, one-mass system is only applicable in the case a rigid coupling with high stiffness is used and there are no flexible elements. Fundamental concept of two-mass systems and their control method are reviewed and explained either in linear or rotary systems.
\end{abstract}

Keywords - Two-mass systems; speed control; position control; literature review.

\section{INTRODUCTION}

In various industrial applications, such as machine tools, measurement machines and semiconductor manufacturing systems mostly are considered as two-mass or multi-mass systems. Its performances on positioning system or speed control depend on the motion accuracy and characteristics of mechanisms and the controllers. The role of the controller is to overcome the mechanical limitations and to utilize their characteristics for high positioning performance. Mechanical elements used in precision positioning systems, such as gear and actuators often have the characteristics, which may degrade the positioning performance, for example, friction of brushes and/or bearings with mechanical contact in motors. Friction will cause steady-state and tracking errors, limit cycles that can slow down of the mechanisms motions [1].

In this paper, the basic concept of the two-mass system is explained in Section 2. This paper also presents the literature review, which is divided into two parts. The first one is focused on the speed control. This is presented in Section 3. On the other hand, position control is reviewed in Section 4. Furthermore, we have divided the position control into linear and rotary system. Lastly, all the discussions are summarized in Section 5.

\section{TwO-MASS SYSTEMS}

In many industrial applications, such as steel rolling mills, flexible robot arms and large space structures, the drive and the load are connected by a coupling shaft which cannot be modelled completely by a rigid body. Often, the simplification as a one-mass system is not appropriate and leads to unsatisfying control performances. Many of these processes can be described as elastic coupled two-mass systems. As a result of costs minimization, the coupling is not stiff but elastic, and includes backlash. Bearings also cause friction which is usually nonlinear. In addition, most parameters are not exactly known, backlash will be larger as time increases, and friction depends on temperature and the lubrication of the bearings. In two-mass systems, if the coupling shaft is not stiff enough, various vibrations are caused by a torsional torque.

Long shafts and spindles lead to elastic torsion, thus, the two-mass systems have the tendency to swing. This can be a problem for standard classical control strategies. There is even the risk of getting an unstable control loop. These vibrations need to be suppressed actively [2]. There are many methods that has been used and studied to control the speed or position ether in linear or rotary systems in order to achieve a good performance. Many researchers have also studied in areas of vibration suppression. In this paper, 
literature review is presented on speed and position control related.

\section{SPEED CONTROL}

Modern drive systems are required to have better and better dynamical properties. They are to track the reference signal with very high accuracy, and to minimize the intermediate phases. For such application, the existing nonlinearities, structural uncertainties, parameter variations, and vibration modes affect the system characteristics. One of the main factors influencing the performances of the drive negatively is finite stiffness of the shaft connecting the motor to the load machine [3].

The conventional control structure based on the proportional integral (PI) speed controller, tuned by the classical symmetric criterion, with a single feedback from the motor speed is not effective in damping the speed oscillations. One of the simplest ways to improve the torsional vibrations ability of the classical structure has been studied based on the modification of the controller parameters [4]. However, this method improves the drive performance only in a limited range of the system parameters. The notch filter (NF) is usually mentioned as a tool ensuring the damping of the oscillations [2]. Rarely a low-pass filter (LPF) or Bi-filter is used. Digital filters can damp the torsional vibration, yet the dynamics of the system may be affected. To improve performances of the classical PI controller, the additional feedback loop from one selected state variable can be used. The additional feedback allows setting the desired value of the damping coefficient, however, the free value of the resonant frequency cannot be achieved simultaneously [5].

The application of the additional feedback from the shaft torque is very common [6]. The design methodology of that system can be divided into two groups. In the first framework, the shaft torque is treated as the disturbance. The simplest approach relies on feeding back the estimated shaft torque to the control structure, with the gain less than one. The more advanced methodology, called resonance ratio control (RRC), is presented in [7]. The system is said to have better damping ability when the ratio of the resonant to antiresonant frequency is a relatively big value. The second framework is based on the modal theory. Parameters of the control structure are calculated by comparing the characteristic equation of the whole system to the desired polynomial. The above control structures are based on the classical cascade compensation schemes.

Since the early $1960 \mathrm{~s}$, a different approach has been developed by using the state-space methodology [8]. However, the industrial application of the new methodology has just started in 1995 such as Kalman filter and linear quadratic (LQ) based speed control [9] and robust outputfeedback $\mathrm{H}_{\infty}$ control [10], where the application of the state controllers for the system with elastic joint is presented. The control structures discussed above allow for the optimal shape of the drive characteristics in the system with constant parameters. Nonetheless, the change of the drive parameters often deteriorates the system performance, and the damping ability of the control structure is reduced.

Recently, the modern control methods have attracted a lot of attention such as the novel nonlinear control structure method for good dynamic performance elastic drives [11]. It is reported to provide a highly damped response. Another approach, observer-based discrete-time sliding mode control [12] which utilize the sliding mode observer to estimate nonmeasurable states of the system. The system is said to be robust against parameter changes and nonlinear elements in the drive. A dynamically generated fuzzy neural [13] network is utilized to damp speed oscillations in a rollingmill drive. It is shown that this approach is more effective than the classical one; furthermore, it is computationally efficient.

The adaptive control has been applied to improve the performance of the drive system with an elastic joint. Two types of the adaptive control can be distinguished [8]. The first one is called the indirect adaptive control. Its general idea is based on the application of the online identification algorithm. The second type of the adaptive control method is called the direct adaptation mechanism. In contrast to the indirect approach, it lacks the identification process. The controller adjusted according to the adaptation law relies on the actually measured values. The adaptive control structure with PI type neuro-fuzzy speed controller is proposed in [14], and good damping of torsional torque and load speed oscillations are obtained. However, in the case of the drive system parameter changes or improper choice of the adaptation law parameters, the tuning properties for different tracks of the reference speed are not optimal, and the required rules number of the adaptive PI type neuro-fuzzy controller must be increased.

\section{POSITION CONTROL}

Position as well as speed control of electrical drives is required for a lot of different industrial processes. Positioning systems that can achieve high accuracy are essential and play important roles in industrial equipments such as machine tools, semiconductor manufacturing systems and robotic systems. Implementation of motion control for positioning system can be classified into two types. The first type is continuous path (CP) control system and the second one is point-to-point (PTP) positioning system. In this paper, literature review on position control for linear and rotary system is presented.

\section{A. Linear System}

In a machining center, work pieces are placed on an XY table. Each axis of the table is linked to a servo motor through a ballscrew which acts like a torsional spring. Since the stiffness of the ballscrew is finite, there exists a torsional displacement resulting in a difference between the position of the motor and the corresponding position of the table. This difference can be significant at a high acceleration and should be compensated to achieve more accurate machining. The demand for high-precision stages has received great attention due to the progress of nano-technology in recent years. Systems to provide long-range and high-precision performance for positioning, tracking and contouring actions have become stringent issues. Among these systems, the ball-screw-driven systems have been widely used in industrial applications and academic research.

There are many control technique that have studied to reduce the tracking error and contour error of a machining 
center. Some of them are feed-forward controllers (FFC) [15], feed-forward friction compensators [16], disturbance observers (DOB) [17], an adaptive robust control (ARC) [18], and model based disturbance attenuators (MBDA) [19]. The FFC compensates for the delay in the response of the control system. The other algorithms are added to a conventional controller to attenuate disturbances more effectively.

Above control algorithms, which are usually implemented using the signal of the encoder attached to a motor, enable the motor to track desired trajectories very well, based on the encoder signal. The tracking performance of the table does not improve very much due to the finite stiffness and backlash of the ballscrew. These mechanical characteristics can cause steady state errors and vibrations of the table's position. In order to overcome these kinds of problems, many methods based on a two-inertia model have been studied in robot arm systems.

The main issues of the studies are vibration suppression and disturbance rejection. [20] studied joint torque feedback using a joint torque sensor in flexible robot arm drive systems, and the relation between vibration suppression and disturbance rejection was analyzed. While the negative joint torque feedback is effective on vibration suppression, it amplifies the effect of disturbances on the end effectors. The positive joint torque feedback can effectively reject disturbances but destabilizes the control system with a flexible joint. The mechanical structure of a flexible arm system is different from an $\mathrm{XY}$ table driven by a long and non-rigid ballscrew, and the purpose of control algorithms of the former is primarily to suppress torsional vibration. Therefore, the negative joint torque feedback is adequate for flexible arm control [21].

For an XY table positioning system, the control based on a measurement of the table's position using a laser interferometer was analyzed by [22]. It showed significant improvements in positioning accuracy, but it seems that the effect of the disturbances on the table, coupled with a nonrigid ballscrew, i.e. torsional displacement, was negligible due to the characteristics of the mechanism. In positioning systems that involve heavy machine tools where the effects of the torsional displacement cannot be negligible, it must be compensated for. If possible, the compensation must be done without using expensive instruments such as a laser interferometer. To develop a simple and effective algorithm to reduce the tracking error of the table's position with motor encoder information only, a torsional displacement feedback control is proposed and analyzed [23] as shown in Fig. 1.

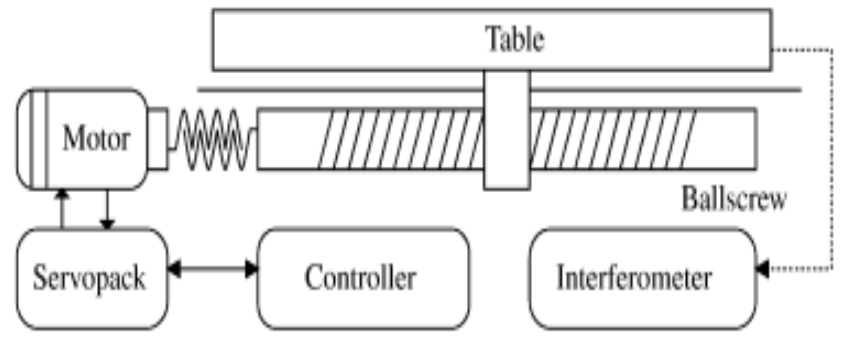

Fig. 1 Table positioning system of a CNC machining center
Sliding mode control (SMC) has gained significant interest in recent years due to its superb characteristics in terms of insensitivity to large parameter variations and its capability in disturbance rejection. The concept of discretetime sliding mode (DSM) [24] was introduced for the purpose of implementing SMC in sampled data systems.

The discrete-time sliding mode control (DSMC) with one step delayed disturbance compensation [25] provides an excellent method for disturbances rejection and chattering attenuation. [26] have shown that the DSMC is able to compensate unknown friction in positioning systems, despite of the complicated characteristics of friction. However, the DSMC cannot be directly applied to the Y-axis ball-screw system, since the controller excessively excites the mechanical resonance of the process [27] as shown in Fig. 2. Many previous researchers have studied the application of the SMC in flexible systems. For example, frequency shaped sliding mode (FSSM) was introduced [28], $\mathrm{H}_{\infty}$ and $\mu$ synthesis based sliding mode control was studied [29].

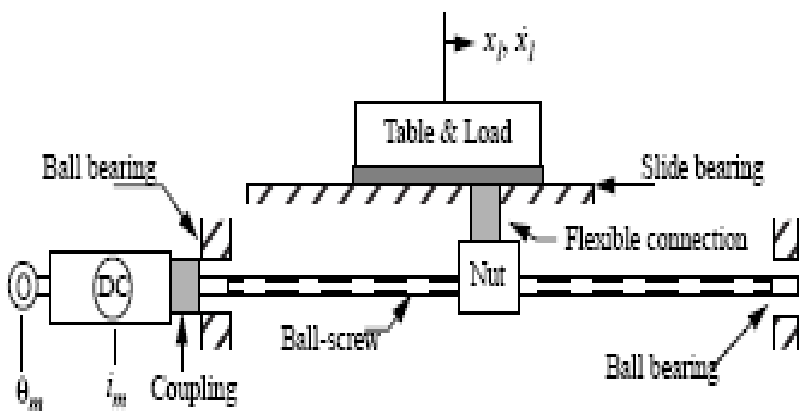

Fig. $2 \mathrm{Y}$-axis of the surface mount robot

These methods in fact, introduce a LPF with an appropriate cut-off frequency into sliding surfaces, thus, high frequency vibrations due to parasitic dynamics as well as their interactions with sliding mode dynamics can be suppressed in the desired frequency band. However, the weakness of such a method is that the transient response may be slowed down to an unsatisfactory degree. To reduce this problem, the combination of a FSSM and a terminal SMC was proposed [30]. Other methods can also be found in the literature, such as sliding mode with perturbation estimation and shaped sliding surface [31], sliding mode with shaped command input [32] and sliding mode with time-varying or nonlinear sliding surface [33]. The extension development of the Nominal Characteristic Trajectory Following (NCTF) as a practical controller has be done experimentally for twomass linear system as shown in Fig. 3. Its structure consists of an nominal characteristic trajectory (NCT) and a PI as a compensator. The positioning performance of the system with friction is examined in comparison with those of the system with conventional proportional integral derivative (PID) controllers. The results prove that the controller has a better positioning performance than those with conventional PID controllers [34]. 


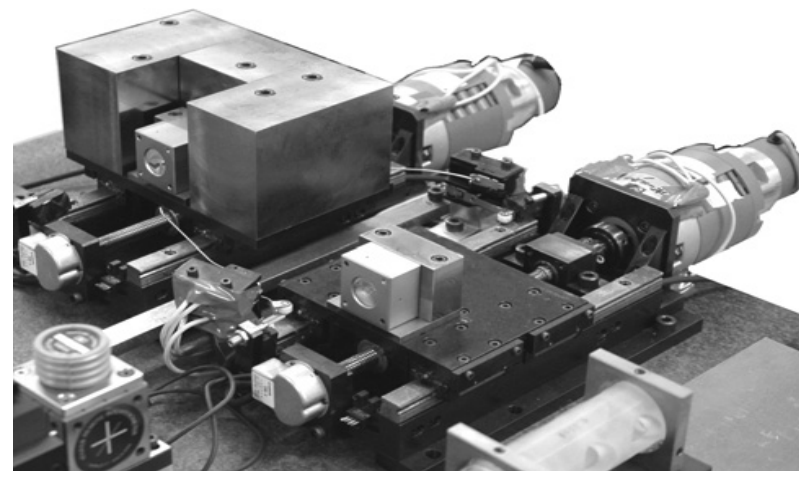

Fig. 3 Two ball screw mechanisms

\section{B. Rotary System}

Electric driving systems with elastic coupling are widely used in various fields such as robots, machine tools, and long shaft driving systems. The dynamic performances of speed and position controlled multi-mass driving system can deteriorate especially due to the elastic coupling, non-linear friction and backlash. This is the reason why researchers look for new solutions for their position control.

One of the most popular control methods in the rotary positioning systems is a PID controller. The feedback gains of the PID controller are usually adjusted for pre-designed load inertia. Many gain tuning methods of PID control such as the ultimate sensitivity method [35], the Ziegler-Nichols tuning method [36], the Cohen-Coon reaction curve methods [37], have been presented. A novel automatic PID tuning also has been proposed that takes both the maximum permissible input and the motor power consumption into consideration. In this PID tuning method, PID gains for a basic one-mass-rigid system are adjusted by the skilful human and the resulted closed loop characteristics are evaluated beforehand [38] as shown in Fig. 4.

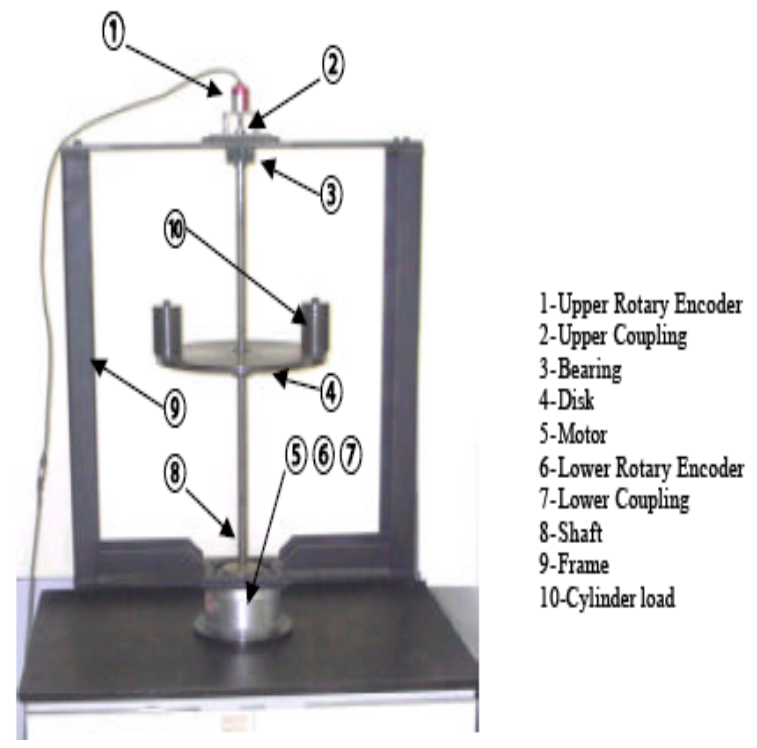

Fig. 4 Experimental system

However, the mechanical coupling between the motor and load machine exhibits unavoidable mechanical imperfection, will cause torsional vibration that may reduce positioning performance response. This has to be carefully considered when high dynamic performances are pursued. Accounting for this imperfection is an involved task, as the structure modeling and the adequate controller design and tuning are becoming very complex.

Based on the simplified linear model of the controlled plant a sliding mode control structure is developed. The proposed sliding mode control structure ensures the best performance in comparison with other classical control structures in the case of an elastic two-mass system. However, tuning problems due to the nonlinear character of the plant could appear when the implementation is going to be performed [39].

Normal PID or state controllers lead to stick-slip effects or oscillations. In addition, most parameters are not exactly known, backlash will get bigger with increasing time, and friction depends on temperature and the lubrication of the bearings. These parameters are important and need to be considered for it implementation in the real time condition. There are controls which can handle nonlinear two-mass systems. But uncertain or even unknown parameters cause problems like loss of quality.

Previously, [40] proposed the combination of friction compensation, a disturbance observer, a position feedback controller, and a feed-forward controller as a general controller structure for high performance robust motion control. Among these four elements, disturbance observer, introduced by [41] and refined by [42] is used to estimate disturbances to make the system robust to plant model uncertainties. However, it is designed based on the linear feedback control theory and it cannot handle discontinuous disturbances such as friction. The disturbance is one of important factors; this makes error or disturbance attenuation which is an objective in the design of most feedback systems. Disturbance observer is added to Kalman filter so that the algorithm may have robustness against the measurements and system errors [43].

A common nonlinear control design via exact linearization has been proposed in [44]. The drive side control has a simple structure. Measurements show good dynamic behaviours and steady-state accuracy even with nonlinear function in the system. But a pre-control is needed to reach steady-state accuracy for the load angle. The precontrol also avoids a tendency to oscillations. The second control is the full-state control. It uses all states and does not need a pre-control. Measurements show good dynamic behaviours of the full-state control. The full state control itself does not reach steady-state accuracy. An additional robust control is needed to improve this. The full-state control is a good basis to put an additional control on top of it. The third presented control result is a full state control with a nonlinear observer. The accuracy of the observed angle is much better than the amount of backlash. The behaviour is similar to the one of the full state control [44].

Long shafts and spindles lead to elastic torsion, thus, the two-mass system has the tendency to swing. This can be a problem for standard control strategies. There is even the risk of getting an unstable control loop. In many cases, these problems are solved by reducing the speed of the control. Additionally, there can be nonlinear parts in the system. The nonlinear characteristics of the mechanical parts, like gears 
and bearings, cannot be neglected. Nonlinear friction of bearings cannot be avoided. Limit cycles and stick-slip effects are known in this context. Many times, the parameters of the system are not known exactly. Especially for the load mass, large variations might exist. The same problem can occur in rotating systems for the load momentum of inertia. The load mass or inertia in transport systems might be unknown. Known self-tuning nonlinear controls show good results for imprecisely known nonlinear plants [45], but time is needed in order to run tuning and retuning algorithms, especially if changes in the system accrue during the operation.

Multiple variations of the system parameters or unstructured parameter uncertainties increase this problem. To handle all the problems mentioned above, an $\mathrm{H}_{\infty}$ control is an appropriate solution. An $\mathrm{H}_{\infty}$ control is a good tool to control linear systems with parameter uncertainties. Once the control is designed no further tuning is needed. The varying parameters and nonlinear parts of the two-mass system are interpreted as unstructured parameter uncertainties for the linear $\mathrm{H}_{\infty}$ control synthesis. On the other hand, the backlash of the two-mass system is explicitly taken into account for the nonlinear observer design [46] as shown in Fig. 5.

In practical applications, an engineer does not need deep knowledge or be an expert in control systems theory while designing controllers. Thus, easiness of controller design process, simplicity of the controller structure and no requirement of exact object model and its parameters are very important and preferable in real applications.

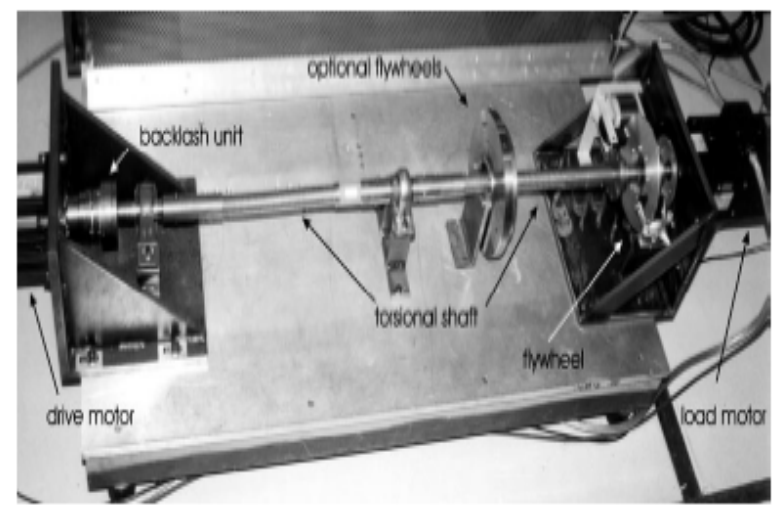

Fig. 5 Experimental setup

To achieve these, NCTF controller for two-mass rotary systems had been proposed as a practical controller for PTP positioning systems in [47] as shown in Fig. 6. The controller design procedure is simple and easily implemented since it is only based on a simple open-loop experiment. In addition, an exact object model and its parameters does not required while designing the controller. Thus, this controller is easy to design, adjustable and understands. The NCTF controller for one-mass system has a better positioning performances and better robustness to parameter variations than the conventional PID controllers [48].

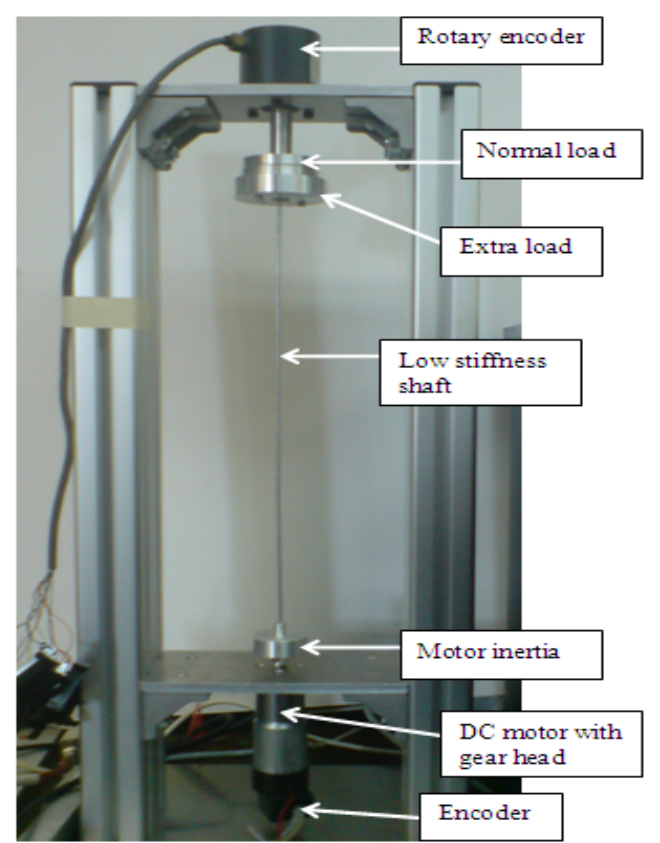

Fig. 6 Lab-scale two-mass rotary systems

\section{CONCLUSIONS}

The two-mass systems are fundamental components in industrial machines such as machine tools, flexible robot arm, measuring machines and semiconductor manufacturing systems. The performances of the machines depend on the positioning systems and speed control of the plant. The literatures review of various controller schemes for speed and position control either in linear or angular system have been discussed and presented. Most of the controllers have been implemented need a deep knowledge in control systems theory which is not suitable for practical applications. Therefore, easiness of controllers design process and simplicity of the controller structure are very important in real applications.

\section{ACKNOWLEDGMENT}

This paper is supported by Malaysia-Japan International Institute of Technology (MJIIT), Universiti Teknologi Malaysia International Campus (UTM IC) Kuala Lumpur, Malaysia.

\section{REFERENCES}

[1] Armstrong-Helouvry B., Dupont P, and De Wit C. (1994). A survey of models, analysis tools and compensation method for the control of machines with friction. Automatica, Vol. 30, No. 7, pp. 1083-1138.

[2] Vukosovic S., N, and Stojic M. R., (1998). Suppression of Torsional Oscillations in a High Performance Speed Servo Drive, IEEE Transaction on Industrial Electronic, pp 108-117, vol. 45, no.1, Technology.

[3] J. Arellano-Padilla, G. M. Asher, and M. Sumner. (2006). Control of an dynamometer for dynamic emulation of mechanical loads with stiff and flexible shafts, IEEE Trans. Ind. Electron., vol. 53, no. 4, pp. $1250-1260$.

[4] G. Zhang and J. Furusho. (2000). Speed control of two-inertia system byPI/PID control, IEEE Trans. Ind. Electron., vol. 47, no. 3, pp. 603609.

[5] K. Szabat and T. Orlowska-Kowalska. (2007). Vibration suppression in two-mass drive system using PI speed controller and additional 
feedbacks - Comparative study, IEEE Trans.Ind. Electron., vol. 54, no. 2, pp. 1193-1206.

[6] M. A. Valenzuela, J. M. Bentley, and R. D. Lorenz, (2005) Evaluation of torsional oscillations in paper machine sections, IEEE Trans. Ind. Appl., vol. 41, no. 2, pp. 493-501.

[7] Y. Hori, H. Sawada, and Y. Chun, (1999). Slow resonance ratio control for vibration suppression and disturbance rejection in torsional system, IEEE Trans. Ind. Electron., vol. 46, no. 1, pp. 162168.

[8] K. Michels, F. Klawonn, R. Kruse, and A. Nürnberger. Fuzzy Control- Fundamentals, Stability and Design of Fuzzy Controllers. New York: Springer, 2006.

[9] J. K. Ji and S. K. Sul, (1995). Kalman filter and LQ based speed controller for torsional vibration suppression in a 2-mass motor drive system, IEEE Trans. Ind. Electron., vol. 42, no. 6, pp. 564-571.

[10] R. Peter, I. Schoeling, and B. Orlik, (2003). Robust output-feedback H1control with a nonlinear observer for a two-mass system, IEEE Trans. Ind. Appl., vol. 39, no. 3, pp. 637-645.

[11] R. Dhaouadi and K. Kubo, (1999). A nonlinear control method for good dynamic performance elastic drives, IEEE Trans. Ind. Electron., vol. 46 , no. 4 , pp. $868-870$.

[12] P. Korondi, H. Hashimoto, and V. Utkin, (1998) Direct torsion control of flexible shaft in an observer-based discrete-time sliding mode, IEEE Trans. Ind. Electron., vol. 45, no. 2, pp. 291-296.

[13] L. Wang and Y. Frayman, (2002). A dynamically generated fuzzy neural network and its application to torsional vibration control of tandem cold rolling mill spindles, Eng. Appl. Artif. Intell., vol. 15, no. 6 , pp. 541-550.

[14] T. Orlowska-Kowalska and K. Szabat, (2007). Control of the drive system with stiff and elastic couplings using adaptive neuro-fuzzy approach, IEEE Trans. Ind. Electron., vol. 54, no. 1, pp. 228-240.

[15] Tomizuka, M. (1987). Zero-phase error tracking algorithm for digital control. ASME, Journal of Dynamic Systems, Measurement and Control, 109(1), 65-68.

[16] Tomizuka, M. (1993). On the compensation of friction forces in precision motion control. Proceedings of the Asia-Pacific Workshop on Advances in Motion Control, pp. 69-74.

[17] Umeno, T., Kaneko, T., \& Hori, Y. (1993). Robust servo system design with two degrees of freedom and its application to novel motion control of robot manipulators. IEEE Transactions on Industrial Electronics, 40(5), pp 473-485.

[18] Yao, B., Al-Majed, M., \& Tomizuka, M. (1997). High-performance robust motion control of machine tools: an adaptive robust control approach and comparative experiments. IEEE/ASME Transactions on Mechatronics, 2(2), pp.63-76.

[19] Choi, B.K., Choi, C.H., \& Lim, H. (1999). Model-based disturbance attenuation for $\mathrm{CNC}$ machining centers in cutting process. IEEE Transactions on Mechatronics, 4(2), pp.157-168.

[20] Zhang, G., Fufusho, J., \& Fajitani, M. (1996). Control of flexiblejoint manipulators using joint torque and acceleration feedback. Proceedings of the 3rd Conference on Motion and Vibration Control, pp. $245-250$.

[21] Zhang, G., \& Furusho, J. (1998). Control of robot arms using joint torque sensors. IEEE Control Systems Magazine, 181, 48-55.

[22] Fischer, M., \& Tomizuka, M. (1996). Application and comparison of alternative position sensors in high-accuracy control of an XY table. Proceedings of the AMC, Mie, pp. 494-499.

[23] Hyuk Lim, Jin-Woo Seo and Chong-Ho Choi. (2000). Torsional displacement compensation in position control for machining centers. Control Engineering Practice 9, pp. 79-87.

[24] Utkin, V. I. (1994). Sliding mode control in discrete time and difference systems. Variable Structure and Lyapunov Control. Alan S.I. Zinober (Ed.) Springer, pp. 87-107.

[25] Su, W.C, S.V. Drakunov \& U. Ozguner (2000). An O (T2) boundary layer in sliding mode for sampled-data systems. IEEE Transactions on Automatic Control 45(3), 482-485.

[26] Li, Y.F, B. Eriksson \& J. Wikander (2000). Discrete-time sliding mode control for linear systems with nonlinear friction. Advances in Variable Structure Systems Analysis, Integration and Applications. Proceedings of the 6th IEEE International Workshop on Variable Structure Systems.
[27] Yu-Feng Li, Jan Wikander. (2002). Discrete-time sliding mode control of a dc motor and ballscrew driven positioning table. 15th Triennial World Congress, Barcelona, Spain.

[28] Young, D. K., V. I. Utkin \& U. Ozguner (1999), A control engineer's guide to sliding mode control. IEEE Transaction on Control System Technology 7(3).

[29] Nonami, K., T. Ito, Y. Kitamura \& K. Iwabuch (1996). Frequencyshaped sliding mode control using $\mathrm{H}_{-}$inf control and $\mathrm{m}$ synthesis theory. 1996 IEEE Workshop on Variable Structure Systems.

[30] Xu, J.X. \& W.J. Cao (2000). Synthesized sliding mode control of a single-like flexible robot. Int. J. control 73(3), 197-209.

[31] Moura, J. T., R. G. Roy \& N. Olgac (1997). Sliding mode contro with perturbation estimation (SMCPE) and frequency shaping sliding surfaces. Transactions of the ASME 119 .

[32] Jalili, N. \& N. Olgac (1998). Time-optimal/sliding mode control implementation for robust tracking of uncertain flexible structures. Mechatronics 8, 121-142.

[33] Hara, S. \& K. Yoshida (1996). Simultaneous optimization of positioning and vibration control using time-varying frequencyshaped criterion function. Control Engineering Practice 4(4), 553-561.

[34] Sato. K, Nakamoto. K, and Shimokohbe. (2004). A Practical Control of Precision Positioning Mechanism with Friction, Precision Engineering, 28, 426-434.

[35] T. Kitamori, (1980). Design Theory of PID Control System, Journal of SICE, Vol. 19, No.4, pp. 382-388.

[36] J. G. Ziegler and N. B. Nichols, (1942). Optimum Setting for Automatic Controllers, Trans. ASME, Vol.64, pp. 759-768.

[37] G. H. Cohen and G. A. Coon, (1953). Theoretical Consideration of Retard Control, Trans. ASME, Vol. 75, pp. 827-834.

[38] Haruhisa Kawasaki and Geng Li. (2001). Automatic PID Tuning Considering Motor Maximum Input and Power Consumption for Servo Control Systems. INTERMAC2001 Joint Technical Conference.

[39] Angela Porumb. (1997). Position Control of an elastic two-mass driving system with backlash and friction, using sliding mode controller. scientific journal, Mechanics, Automatic, Control and Robotics Vol.2, No 7, pp. 285 -290.

[40] Bin Yao and Masayoshi Tomizuka, (1997). High Performance Robust Motion Control of Machine Tools: An Adaptive Robust Control Approach and Comparative Experiments, Proc. ACC., pp. 2754-2758.

[41] Ohnishi. K., Shibata. M. and Murakami. T.,(1996). Motion Control for Advanced Mechatronics, IEEElAMSE Trans. on Mechatronics, Vol. I, No.1, pp. 56-67.

[42] Umeno and Hori, (1991). Robust Speed Control of DC Servomotors using Modern Two degrees-of-Freedom Controller Design, IEEE Trans. on IE, Vo1.38, No.5, pp.363-368.

[43] Doo- Jin Shin and Uk-Youl Huh. (2000). Robust Motion Controller Design for Servo System with 2 Mass Characteristics. AMC2000. Nagoya. Pp. 423-426.

[44] I. Scholing, B. Orlik. (2000). Control of a Nonlinear Two-Mass System with Uncertain Parameters and Unknown States. IEEE. pp.1096-1103.

[45] D. Schroder, (2000), Intelligent Observer and Control Design for Nonlinear Systems Springer.

[46] Karsten Peter, Ingo Schöling, and Bernd Orlik. (2003). Robust Output-Feedback H Control With a Nonlinear Observer for a TwoMass System. IEEE Trans.Ind. Applications, vol. 39, no. 3, pp. $637-$ 644

[47] Fitri M.Y, Wahyudi and R.Akmeliawati, Improved NCTF Control Method for a Two Mass Point to Point Positioning System, Proceedings of the 2010 IEEE $3^{\text {rd }}$ International Conference on Intelligent and Advanced systems (icias 2010), Kuala Lumpur, Jun 2010 .

[48] Mohd Fitri Mohd Yakub, and R.Akmeliawati, Performance Improvement of Improved Practical Control Method for Two-Mass PTP Positioning Systems in the Presence of Actuator Saturation, Proceedings of the 2011 IEEE Applied Power Electronics Colloquium (IAPEC2011) 18-19 April 2011, Johor Baru. 